DOI:

УДК 681.04

Ю.Д. Поліський, к.т.н, polissky477@gmail.com

НДІ автоматизації чорної металургії, м. Дніпро

\title{
МЕТОДИ РЕАЛІЗАЦІЇ ДЕЯКИХ СКЛАДНИХ ОПЕРАЦІЙ У СИСТЕМІ ЗАЛИШКОВИХ КЛАСІВ
}

\author{
В роботі досліджені системи залишкових класів з можливістю реалізаиії складних опе- \\ рачій попарного і групового порівняння чисел. \\ Ключові слова: залишкові класи; проблемні операчії; модулі; порівняння чисел.
}

In this work the systems of residual classes with the possibility of realization of complex operations of pairwise and group comparison of numbers are researched.

Keywords: residual classes; problem operations; modules; comparison of numbers.

\section{Постановка проблеми}

Функціонування систем управління завжди включає порівняння станів керованих об'єктів, що оцінюються відповідними значеннями чисел. В одних випадках операція порівняння являє собою зіставлення значень двох чисел (попарне порівняння) і перевірку наявності тієї чи іншої ознаки у результату. В інших випадках при порівнянні аналізується група чисел (групове порівняння), а результат являє собою найбільше або найменше значення, ознаку положення чисел по відношенню до граничних значень або до деякого фіксованого значення або довжину диапазону значень аналізованих чисел. До обчислювальних структур, за допомогою яких виконуються вищезгадані операції порівняння чисел, постійно пред'являються вимоги підвищення швидкодії. Застосування системи залишкових класів (СЗК) дозволяє підвищити продуктивність таких систем.

При істотних перевагах виконання операцій порівняння чисел у СЗК [1] виникають певні труднощі їх реалізації в цій системі, що потребують подальших досліджень.

\section{Аналіз останніх досліджень і публікацій}

Вирішенню складностей при реалізації операцій порівняння чисел у СЗК присвячений ряд публікацій $[2,3,4,5]$. При значних досягненнях на теперішній час у зіставленні з початковими рішеннями, що викладені у класичній фундаментальній монографії [1], залишаються певні вимоги до деяких показників, зокрема, швидкодії операцій порівняння чисел.

\section{Формулювання мети дослідження}

Метою дослідження $є$ аналітичний розгляд СЗК для реалізації базових складних операцій попарного і групового порівняння чисел.

\section{Виклад основного матеріалу}

Розвиток сучасних інформаційних і керуючих систем пов'язаний із застосуванням нових принципів представлення даних, зокрема, в системі залишкових класів. Під СЗК розуміють систему числення, в якій довільне число $N$ представляється у вигляді набору найменших невід'ємних залишків по модулях $m_{1}, m_{2}, \ldots, m_{n}$, тобто $N=\left(\alpha_{1}, \alpha_{2}, \ldots, \alpha_{n}\right)$. Тут $\alpha_{i}=N\left(\bmod m_{i}\right)$. При цьому, якщо числа $m_{i}$ попарно взаємно прості, то такому представленню відповідає тільки одне число $N$ диапазону $[0, M)$, де $M=m_{1} m_{2} \ldots m_{n}$.

Якщо системою модулів поліадичного коду також $\epsilon$ система $m_{1}, m_{2}, \ldots, m_{n}$, число $N$ в поліадичному коді представляється у вигляді

$$
N=\pi_{1}+\pi_{2} m_{1}+\ldots+\pi_{i} m_{1} m_{2} \ldots m_{i-1}+\ldots+\pi_{n-1} m_{1} m_{2} \ldots m_{n-2}+\pi_{n} m_{1} m_{2} \ldots m_{n-1} \text {, де } \pi_{i} \text { 一 по- }
$$

зиційна характеристика, $0 \leq \pi_{i} \leq m_{i}-1$. 
Отже, при попарному порівнянні чисел $N_{1}=\left(\alpha_{1}, \alpha_{2}, \ldots, \alpha_{n}\right)$ та $N_{1}=\left(\beta_{1}, \beta_{2}, \ldots, \beta_{n}\right)$ необхідно визначити результат $R=\left\{\begin{array}{l}R_{1} \cdot N_{1}>N_{2} \\ R_{2} \cdot N_{1}<N_{2}\end{array}\right.$.

При груповому порівнянні чисел

$$
\begin{aligned}
& N_{2}=\left(\alpha_{1}^{2}, \alpha_{2}^{2}, \ldots, \alpha_{i}^{2}, \ldots, \alpha_{n}^{2}\right), \\
& \ldots \ldots \ldots \ldots \ldots \ldots \ldots . \ldots . \\
& N_{j}=\left(\alpha_{1}^{j}, \alpha_{2}^{j}, \ldots, \alpha_{i}^{j}, \ldots, \alpha_{n}^{j}\right), \\
& \ldots \ldots \ldots \ldots \ldots \ldots \ldots . \ldots . \ldots . \\
& N_{k}=\left(\alpha_{1}^{k}, \alpha_{2}^{k}, \ldots, \alpha_{i}^{k}, \ldots, \alpha_{n}^{k}\right)
\end{aligned}
$$

вирішуються наступні завдання:

- визначення максимального і мінімального чисел групи,

- визначення довжини диапазону групи чисел,

- визначення положення чисел групи по відношенню до деякого фіксованого числа,

- визначення чисел, що лежать всередині деякого піддиапазону,

- визначення числа, найближчого до заданого числа,

- визначення числа, найближчого більшого до заданого і найближчого меншого до заданого числа.

Дослідження операцій порівняння чисел в СЗК показали, що все розмаїття рішень може бути отримано на підставі трьох підходів. По першому для кожного з порівнюваних чисел обчислюються позиційні характеристики, після чого здійснюється порозрядне порівняння цих характеристик традиційними методами порівняння. Другий підхід використовує визначення приналежності чисел $N_{1}, N_{2}$ та їх різниці $\Delta=N_{1}-N_{2}$ до верхньої або нижньої половині диапазону чисел. Якщо $N_{1}$ та $N_{2}$ належать до різних половин диапазону, то більшим (меншим) є число верхньої (нижньої) половини. Якщо ж $N_{1}$ та $N_{2}$ належать до однієї половини, то при належності $\Delta$ до верхньої половини диапазону $N_{1}>N_{2}$. Третій підхід був вперше запропонований в [6] i розвинений в роботі [7]. Підхід заснований на складанні для кожного з чисел різниць $\widetilde{\alpha}_{i}=\left(\alpha_{i}-\alpha_{n}\right)\left(\bmod m_{i}\right), i=1,2, \ldots, n-1$, в результаті чого весь диапазон $M$ виявляється розбитим на $K=\frac{M}{m_{n}}$ піддиапазонів довжини $m_{n}$, всередині кожного з яких значення $\widetilde{\alpha}_{i}$ однакові.

Далі розглянемо третій підхід до вирішення завдань попарного і групового порівняння чисел на основі [6] та для зіставлення з використанням вперше отриманого в [8] ефективного вирішення, заснованого на одночасному поданні чисел в прямому і зворотному кодах з вибором активного представлення на кожній ітерації.

Згідно 3 [8] $\bar{N}=\left(\bar{\alpha}_{1}, \bar{\alpha}_{2}, \ldots, \bar{\alpha}_{n}\right)$ є представленням числа $N=\left(\alpha_{1}, \alpha_{2}, \ldots, \alpha_{n}\right)$ в зворотному коді, де $\bar{\alpha}_{i}=\left(m_{i}-1\right)-\alpha_{i}$.

Для розгляду роботи необхідні наведені нижче табл. 1, 2, 3, 4 .

Табл. 1 ілюструє виконання за [6] операції попарного порівняння чисел $N_{1}=1183=$ $(1,1,3,0,6)$ та $N_{2}=752=(0,2,2,3,4)$ по модулях $2,3,5,7,11$. Оскільки в кінцевому результаті $\widetilde{N}_{2}=0<\widetilde{N}_{1}=3$, то $N_{2}=752<N_{1}=1183$. При цьому результат досягається за $T_{1}^{n n}=7$ ітерацій.

Табл. 2 ілюструє виконання за [6] операції попарного порівняння чисел $N_{1}=1183=$ $(1,1,3,0,6)$ та $N_{2}=752=(0,2,2,3,4)$ по тих же модулях $2,3,5,7,11$ з використанням одночасного представлення чисел в прямому і зворотному кодах. Оскільки в кінцевому результаті $\widetilde{N}_{2}=0<\widetilde{N}_{1}=5$, то $N_{2}=752<N_{1}=1183$. Тут результат досягається за $T_{2}^{n n}=5$ ітерацій, тобто виграш у швидкодії $\theta=\frac{7}{5}=1,4$ рази. 
Таблиия 1

\begin{tabular}{|c|c|c|c|c|c|c|}
\hline \multirow[t]{3}{*}{ Числа } & \multicolumn{5}{|c|}{ Модулі } & \multirow{3}{*}{ Ітерація 1} \\
\hline & 2 & 3 & 5 & 7 & 11 & \\
\hline & \multicolumn{5}{|c|}{ Залишки } & \\
\hline$N_{1}=\mathbf{1 1 8 3}$ & 1 & 1 & 3 & 0 & 6 & -6 \\
\hline$N_{2}=752$ & 0 & 2 & 2 & 3 & 4 & -4 \\
\hline \multirow[t]{3}{*}{ Числа } & \multicolumn{5}{|c|}{ Модулі } & \multirow{3}{*}{ Ітерація 2} \\
\hline & 2 & \begin{tabular}{|l|}
3 \\
\end{tabular} & 5 & 7 & 11 & \\
\hline & \multicolumn{5}{|c|}{ Залишки } & \\
\hline$\tilde{N}_{1}==1177$ & 1 & 1 & 2 & 1 & 0 & $: 11$ \\
\hline$\tilde{N}_{2}=748$ & 0 & 1 & 3 & 6 & 0 & $: 11$ \\
\hline \multirow[t]{3}{*}{ Числа } & \multicolumn{5}{|c|}{ Модулі } & \multirow{3}{*}{ Ітерація 3} \\
\hline & 2 & 3 & 5 & 7 & 11 & \\
\hline & \multicolumn{5}{|c|}{ Залишки } & \\
\hline$\tilde{N}_{1}=\mathbf{1 0 7}$ & 1 & 2 & 2 & 2 & $==$ & -2 \\
\hline$\tilde{N}_{2}=\mathbf{6 8}$ & 0 & 2 & 3 & 5 & $==$ & -5 \\
\hline \multirow[t]{3}{*}{ Числа } & \multicolumn{5}{|c|}{ Модулі } & \multirow{3}{*}{ Ітерація 4} \\
\hline & 2 & \begin{tabular}{|l|}
3 \\
\end{tabular} & 5 & 7 & 11 & \\
\hline & \multicolumn{5}{|c|}{ Залишки } & \\
\hline$\tilde{N}_{1}=\mathbf{1 0 5}$ & 1 & 0 & 0 & 0 & $==$ & $: 7$ \\
\hline$\tilde{N}_{2}=63$ & 1 & 0 & 3 & 0 & $==$ & $: 7$ \\
\hline \multirow[t]{3}{*}{ Числа } & \multicolumn{5}{|c|}{ Модулі } & \multirow{3}{*}{ Ітерація 5} \\
\hline & 2 & 3 & 5 & 7 & 11 & \\
\hline & \multicolumn{5}{|c|}{ Залишки } & \\
\hline$\tilde{N}_{1}=15$ & 1 & 0 & 0 & $==$ & $==$ & 0 \\
\hline$\tilde{N}_{2}=\mathbf{9}$ & 1 & 0 & 4 & $==$ & $==$ & -4 \\
\hline \multirow[t]{3}{*}{ Числа } & \multicolumn{5}{|c|}{ Модулі } & \multirow{3}{*}{ Ітерація 6} \\
\hline & 2 & 3 & 5 & 7 & 11 & \\
\hline & \multicolumn{5}{|c|}{ Залишки } & \\
\hline$\tilde{N}_{1}=15$ & 1 & 0 & 0 & $==$ & $==$ & $: 5$ \\
\hline$\tilde{N}_{2}=\mathbf{5}$ & 1 & 2 & 0 & $==$ & $==$ & $: 5$ \\
\hline \multirow[t]{3}{*}{ Числа } & \multicolumn{5}{|c|}{ Модулі } & \\
\hline & 2 & 3 & 5 & 7 & 11 & Ітерація 7 \\
\hline & & & Зал & шки & & \\
\hline$\tilde{N}_{1}=\mathbf{3}$ & 1 & 0 & $==$ & $==$ & $==$ & 0 \\
\hline$\tilde{N}_{2}=\mathbf{1}$ & 1 & 1 & $==$ & $==$ & $==$ & -1 \\
\hline Числа & & & & улі & & \\
\hline & 2 & 3 & 5 & 7 & 11 & \\
\hline & & & Зал & шки & & \\
\hline$\tilde{N}_{1}=\mathbf{3}$ & 1 & 0 & $==$ & $==$ & $==$ & \\
\hline$\tilde{N}_{2}=\mathbf{0}$ & 0 & 0 & $==$ & $==$ & $==$ & \\
\hline
\end{tabular}


Табличя 2

\begin{tabular}{|c|c|c|c|c|c|c|c|c|c|c|c|c|c|}
\hline \multirow{3}{*}{\multicolumn{2}{|c|}{ Числа }} & \multicolumn{4}{|c|}{ Модулі } & Ітерація 1 & \multirow[t]{3}{*}{ Числа } & \multicolumn{5}{|c|}{$\begin{array}{l}\text { Мо- } \\
\text { дулі }\end{array}$} & $\begin{array}{l}\text { Ітерація } \\
1\end{array}$ \\
\hline & & \begin{tabular}{l|l}
2 & 3 \\
\end{tabular} & 5 & 7 & 11 & & & 2 & 3 & & \begin{tabular}{l|l}
5 & 7 \\
\end{tabular} & 11 & \\
\hline & & \multicolumn{4}{|c|}{ Залишки } & & & \multicolumn{5}{|c|}{ Залишки } & \\
\hline \multicolumn{2}{|l|}{$N_{1}=1183$} & 11 & 3 & 0 & 6 & -6 & $\bar{N}_{1}=1126$ & 0 & 1 & 1 & 6 & 4 & -4 \\
\hline \multicolumn{2}{|l|}{$N_{2}=752$} & $\begin{array}{ll}0 & 2\end{array}$ & 2 & 3 & 4 & -4 & $\bar{N}_{2}=1557$ & 1 & 0 & 2 & 3 & 6 & -6 \\
\hline & & & & & & & \multirow[t]{3}{*}{ Числа } & \multicolumn{5}{|c|}{ Модулі } & \multirow{3}{*}{$\begin{array}{l}\text { Ітерація } \\
2\end{array}$} \\
\hline & & & & & & & & 2 & 3 & 5 & 7 & 11 & \\
\hline & & & & & & & & \multicolumn{5}{|c|}{ Залишки } & \\
\hline & & & & & & & $\tilde{N}_{1}=1122$ & 0 & 0 & 2 & 2 & 0 & $:(3 * 11)$ \\
\hline & & & & & & & $\tilde{N}_{2}=1551$ & 1 & 0 & 1 & 4 & 0 & $:(3 * 11)$ \\
\hline Числа & \multicolumn{5}{|c|}{ Модулі } & \multirow{3}{*}{ Ітерація 3} & Числа & \multicolumn{5}{|c|}{ Модулі } & \\
\hline & 2 & 3 & 5 & 7 & 11 & & & 2 & 3 & 5 & 7 & 11 & \\
\hline & \multicolumn{5}{|c|}{ Залишки } & & & \multicolumn{5}{|c|}{ Залишки } & \\
\hline$\tilde{N}_{1}=35$ & 1 & $=$ & 0 & 0 & $=$ & 0 & $\tilde{N}_{1}=\mathbf{3 4}$ & 0 & $=$ & 4 & 6 & $=$ & -6 \\
\hline$\tilde{N}_{2}=\mathbf{2 2}$ & 0 & $=$ & 2 & 1 & $=$ & -1 & $\tilde{N}_{2}=\mathbf{4 7}$ & 1 & $=$ & 2 & 5 & $=$ & -5 \\
\hline \multirow[t]{3}{*}{ Числа } & \multicolumn{5}{|c|}{ Модулі } & \multirow{3}{*}{ Ітерація 4} & & & & & & & \\
\hline & 2 & 3 & 5 & 7 & 11 & & & & & & & & \\
\hline & \multicolumn{5}{|c|}{ Залишки } & & & & & & & & \\
\hline$\tilde{N}_{1}=\mathbf{3 5}$ & 1 & $=$ & 0 & 0 & $=$ & $: 7$ & & & & & & & \\
\hline$\tilde{N}_{2}=\mathbf{2 1}$ & 1 & $=$ & 1 & 0 & $=$ & $: 7$ & & & & & & & \\
\hline \multirow[t]{3}{*}{ Числа } & \multicolumn{5}{|c|}{ Модулі } & \multirow{3}{*}{ Ітерація 5} & & & & & & & \\
\hline & 2 & 3 & 5 & 7 & 11 & & & & & & & & \\
\hline & \multicolumn{5}{|c|}{ Залишки } & & & & & & & & \\
\hline$\tilde{N}_{1}=\mathbf{5}$ & 1 & $=$ & 0 & $=$ & $=$ & 0 & & & & & & & \\
\hline$\tilde{N}_{2}=\mathbf{3}$ & 1 & $=$ & 3 & $=$ & $=$ & -3 & & & & & & & \\
\hline \multirow[t]{3}{*}{ Числа } & \multicolumn{5}{|c|}{ Модулі } & & & & & & & & \\
\hline & 2 & 3 & 5 & 7 & 11 & & & & & & & & \\
\hline & & & али & шкІ & & & & & & & & & \\
\hline$\tilde{N}_{1}=\mathbf{5}$ & 1 & $=$ & 0 & $=$ & $=$ & & & & & & & & \\
\hline$\tilde{N}_{2}=\mathbf{0}$ & 0 & $=$ & 0 & $=$ & $=$ & & & & & & & & \\
\hline
\end{tabular}

Табл. 3 ілюструє виконання за [6] операції групового порівняння чисел, наприклад, чисел $\quad N_{1}=1808=(0,2,3,2,4), \quad N_{2}=1310=(0,2,0,1,1), \quad N_{3}=658=(0,1,3,0,9)$, $N_{4}=1315=(1,1,0,6,6)$ по тих же модулях $2,3,5,7,11$. Оскільки в кінцевому результаті $\tilde{N}_{3}=0<\tilde{N}_{2}=3$, то $N_{3}=\min \left\{N_{1}, N_{2}, N_{3}, N_{4}\right\}$. При цьому результат досягається за $T_{3}^{n n}=7$ ітерацій. 
Таблицуя 3

\begin{tabular}{|c|c|c|c|c|c|c|}
\hline \multirow[t]{3}{*}{ Числа } & \multicolumn{5}{|c|}{ Модулі } & \multirow{3}{*}{ Ітерація 1} \\
\hline & 2 & 3 & 5 & 7 & 11 & \\
\hline & \multicolumn{5}{|c|}{ Залишки } & \\
\hline$N_{1}=\mathbf{1 8 0 8}$ & 0 & 2 & 3 & 2 & 4 & -4 \\
\hline$N_{2}=1310$ & 0 & 2 & 0 & 1 & 1 & -1 \\
\hline$N_{3}=\mathbf{6 5 8}$ & 0 & 1 & 3 & 0 & 9 & -9 \\
\hline$N_{4}=1315$ & 1 & 1 & 0 & 6 & 6 & -6 \\
\hline \multirow[t]{3}{*}{ Числа } & \multicolumn{5}{|c|}{ Модулі } & \multirow{3}{*}{ Ітерація 2} \\
\hline & 2 & 3 & 5 & 7 & 11 & \\
\hline & \multicolumn{5}{|c|}{ Залишки } & \\
\hline$\tilde{N}_{1}=1804$ & 0 & 1 & 4 & 5 & 0 & $: 11$ \\
\hline$\tilde{N}_{2}=\mathbf{1 3 0 9}$ & 1 & 1 & 4 & 0 & 0 & $: 11$ \\
\hline$\tilde{N}_{3}=649$ & 1 & 1 & 4 & 5 & 0 & $: 11$ \\
\hline \multirow[t]{3}{*}{ Числа } & \multicolumn{5}{|c|}{ Модулі } & \multirow{3}{*}{ Ітерація 3} \\
\hline & 2 & 3 & 5 & 7 & 11 & \\
\hline & & & & & & \\
\hline$\tilde{N}_{1}=164$ & 0 & 2 & 4 & 3 & $==$ & -3 \\
\hline$\tilde{N}_{2}=119$ & 1 & 2 & 4 & 0 & $==$ & 0 \\
\hline$\tilde{N}_{3}=\mathbf{5 9}$ & 1 & 2 & 4 & 3 & $==$ & -3 \\
\hline \multirow[t]{3}{*}{ Числа } & \multicolumn{5}{|c|}{ Модулі } & \multirow{3}{*}{ Ітерація 4} \\
\hline & 2 & 3 & 5 & 7 & 11 & \\
\hline & & & & & & \\
\hline$\tilde{N}_{1}=\mathbf{1 6 1}$ & 1 & 2 & 1 & 0 & $==$ & $: 7$ \\
\hline$\tilde{N}_{2}=119$ & 1 & 2 & 4 & 0 & $==$ & $: 7$ \\
\hline$\tilde{N}_{3}=56$ & 0 & 2 & 1 & 0 & $==$ & $: 7$ \\
\hline \multirow[t]{3}{*}{ Числа } & \multicolumn{5}{|c|}{ Модулі } & \multirow{3}{*}{ Ітерація 5} \\
\hline & 2 & 3 & 5 & 7 & 11 & \\
\hline & & & & & & \\
\hline$\tilde{N}_{1}=\mathbf{2 3}$ & 1 & 2 & 3 & $==$ & $==$ & -3 \\
\hline$\tilde{N}_{2}=\mathbf{1 7}$ & 1 & 2 & 2 & $==$ & $==$ & -2 \\
\hline$\tilde{N}_{3}=\mathbf{8}$ & 0 & 2 & 3 & $==$ & $==$ & -3 \\
\hline \multirow[t]{3}{*}{ Числа } & \multicolumn{5}{|c|}{ Модулі } & \multirow{3}{*}{ Ітерація 6} \\
\hline & 2 & 3 & 5 & 7 & 11 & \\
\hline & \multicolumn{5}{|c|}{ Залишки } & \\
\hline$\tilde{N}_{1}=\mathbf{2 0}$ & 0 & 2 & 0 & $==$ & $==$ & $: 5$ \\
\hline$\tilde{N}_{2}=15$ & 1 & 0 & 0 & $==$ & $==$ & $: 5$ \\
\hline$\tilde{N}_{3}=\mathbf{5}$ & 1 & 2 & 0 & $==$ & $==$ & $: 5$ \\
\hline
\end{tabular}


Закінчення таблиці 3

\begin{tabular}{|c|c|c|c|c|c|c|}
\hline \multirow[t]{3}{*}{ Числа } & \multicolumn{5}{|c|}{ Модулі } & \multirow{3}{*}{ Ітерація 7} \\
\hline & 2 & 3 & 5 & 7 & 11 & \\
\hline & \multicolumn{5}{|c|}{ Залишки } & \\
\hline$\tilde{N}_{1}=\mathbf{4}$ & 0 & 1 & $==$ & $==$ & $==$ & -1 \\
\hline$\tilde{N}_{2}=\mathbf{3}$ & 1 & 0 & $==$ & $==$ & $==$ & 0 \\
\hline$\tilde{N}_{3}=\mathbf{1}$ & 1 & 1 & $==$ & $==$ & $==$ & -1 \\
\hline \multirow[t]{3}{*}{ Числа } & \multicolumn{5}{|c|}{ Модулі } & \\
\hline & 2 & 3 & 5 & 7 & 11 & \\
\hline & \multicolumn{5}{|c|}{ Залишки } & \\
\hline$\tilde{N}_{1}=\mathbf{3}$ & 1 & 0 & $==$ & $==$ & $==$ & \\
\hline$\tilde{N}_{2}=\mathbf{3}$ & 1 & 0 & $==$ & $==$ & $==$ & \\
\hline$\tilde{N}_{3}=\mathbf{0}$ & 0 & 0 & $==$ & $==$ & $==$ & \\
\hline
\end{tabular}

Табл. 4 ілюструє виконання за [6] операції групового порівняння тих же чисел $N_{1}=1808=(0,2,3,2,4), \quad N_{2}=1310=(0,2,0,1,1), \quad N_{3}=658=(0,1,3,0,9)$, $N_{4}=1315=(1,1,0,6,6)$ по тих же модулях 2, 3, 5, 7, 11 з використанням одночасного представлення чисел в прямому і зворотному кодах. Оскільки в кінцевому результаті $\widetilde{N}_{3}=0<\widetilde{N}_{2}=7$, то $N_{3}=\min \left\{N_{1}, N_{2}, N_{3}, N_{4}\right\}$. При цьому результат досягається за $T_{4}^{n n}=2$ ітерації, тобто виграш у швидкодії $\theta=\frac{7}{2}=3,5$ рази.

Отже, розглянуто отримання мінімального $N_{\min }$ та максимального $N_{\max }$ чисел групи. Вирішення решти завдань групового порівняння чисел здійснюється наступним чином.

Довжина $L_{\Delta}$ диапазону $\Delta=\left[N_{\max }, N_{\min }\right]$ групи чисел визначається, як $L_{\Delta}=N_{\max }-N_{\min }$.

Визначення положення чисел групи по відношенню до деякого фіксованого числа полягає в одночасному порівнянні кожного з чисел групи з даним числом і отриманні результату в залежності від співвідношення $N>N_{\phi}, N<N_{\phi}$ або $N=N_{\phi}$.

Для визначення чисел, що лежать всередині деякого піддиапазону $\Delta_{t} \in \Delta$, заданого фіксованими граничними значеннями $N_{t} \delta_{1}$ та $N_{t} \delta_{2}$, виконується одночасне порівняння кожного 3 чисел групи з граничними значеннями, а також порівняння $N_{t \delta_{1}}{ }^{3} N_{t \delta_{2}}$. Тоді рішенням завдання є всі числа групи $\left\{N^{1}, N^{2}, N^{j}, \ldots, N^{k}\right\}$, що задовольняють умові $N_{t \delta_{1}} \geq N^{J} \geq N_{t \delta_{2}}$.

Визначення числа, найближчого до заданого числа, включає дві ітерації. На першій виконується попарне порівняння кожного числа групи з заданим числом для визначення більшого $N_{\max }^{j}=\max \left\{N_{c}, N^{J}\right\}$ і меншого $N_{\min }^{j}=\min \left\{N_{c}, N^{J}\right\}$ в кожній парі і визначаються значення $\delta^{j}=N_{\max }^{j}-N_{\min }^{j}, j=1,2, \ldots, k$. На другий ітерації виконується групове порівняння чисел для отримання $\delta_{\min }^{j}=\min \left\{\delta^{1}, \delta^{2}, \ldots, \delta^{j}, \ldots, \delta^{k}\right\}$. 
Таблиия 4

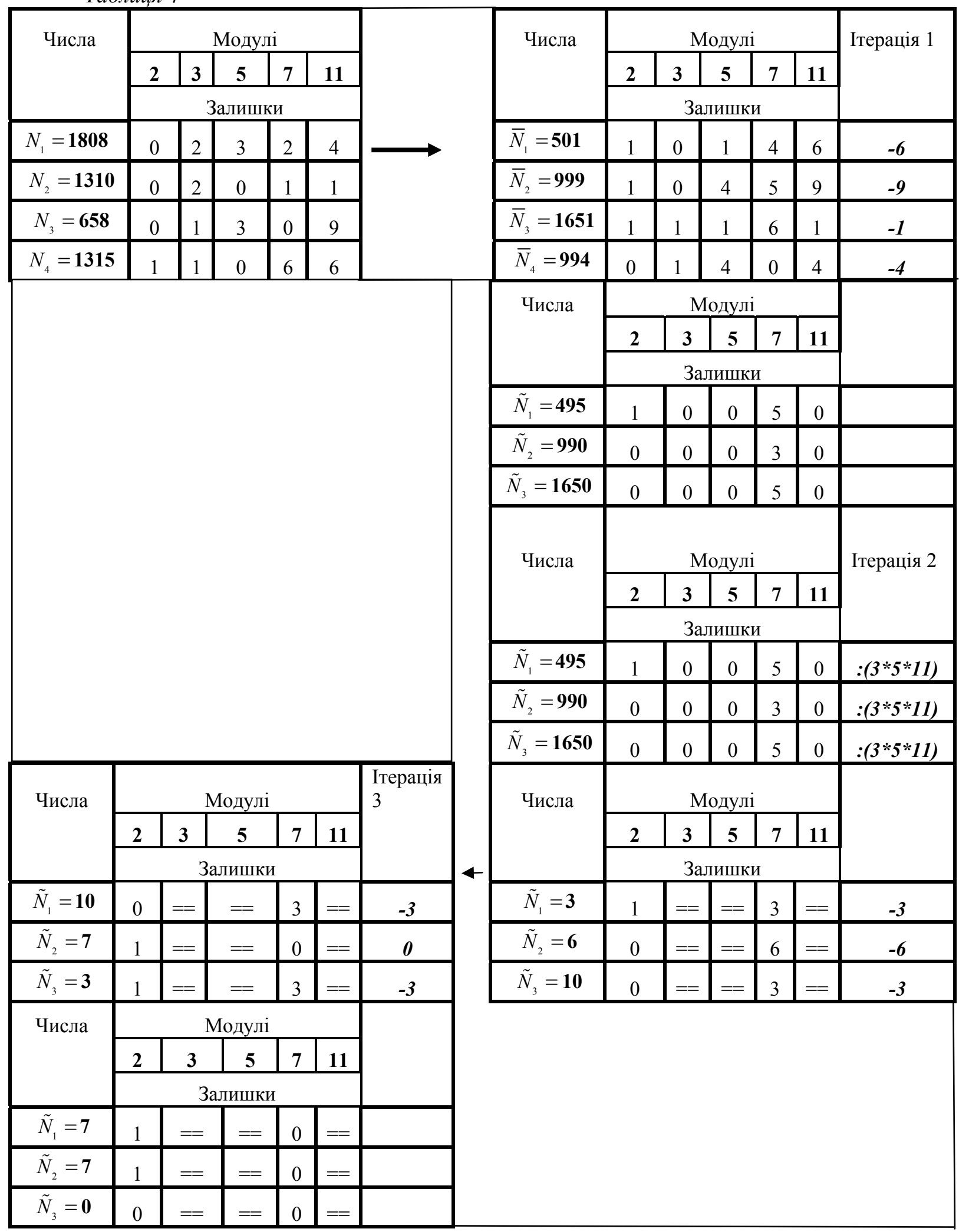

Визначення числа, найближчого більшого до заданого і найближчого меншого до заданого числа здійснюється також за дві ітерації. Перша ітерація аналогічна першій ітерації попереднього алгоритму. На другій ітерації виконується групове порівняння чисел, більших за зада- 
не, для отримання $\delta_{\sigma_{\text {min }}}^{j}=\min \left\{\delta_{\sigma}^{1}, \delta_{\sigma}^{2}, \ldots, \delta_{\sigma}^{j}, \ldots, \delta_{\sigma}^{k}\right\}$, і групове порівняння чисел, менших за задане, для отримання $\delta_{\mathrm{M}_{\min }}^{j}=\min \left\{\delta_{\mathrm{M}}^{1}, \delta_{\mathrm{M}}^{2}, \ldots, \delta_{\mathrm{M}}^{j}, \ldots, \delta_{\mathrm{M}}^{k}\right\}$.

\title{
Висновки
}

Розглянуті методи розв'язання задач попарного та групового порівняння чисел, представлених в системі залишкових класів. Показано, що проаналізовані методи забезпечують отримання комплексу результатів порівняння чисел. На основі запропонованих підходів досягається підвищення швидкодії виконання операцій порівняння. Представляється доцільним застосувати запропоновані підходи в якості перспективного напрямку досліджень задач порівняння чисел в системі залишкових класів.

\section{Список використаної літератури}

1. Акушский И.Я., Юдицкий Д.И. Машинная арифметика в остаточных классах. [Текст] / И.Я Акушский., Д.И. Юдицкий. - М.: Советское радио, 1968. - 440 с.

2. Поліський Ю.Д.,Методи порівняння чисел у системі залишкових класів. Науковий вісник НГУ. - 2007. №1. - С. 63-66.

3. Исупов К.С. Об одном алгоритме сравнения чисел в системе остаточных классов // Вестн. Астрахан. гос. техн. ун-та. Сер. управление, вычисл. техн. информ., 2014, №3, С. 40-49.

4. Кнут,Д. Искусство программирования / Д.Кнут. - Москва: Диалектика-Вильямс, 2013. - 832 с.

5. Полисский Ю.Д. Сравнение чисел в системе остаточных классов. / Труды Юбилейной Международной научно-технической конференции «50 лет модулярной арифметики», Россия, Москва, Зеленоград, 23-25 ноября 2005, издательство МИЭТ, с. 274-290.

6. Факторович М.Г., Полисский Ю.Д. Устройство для сравнения чисел, выраженных в системе остаточных классов. Авт. свид. СССР №608155 М.Клㅡ G 06 F 7/04, 1978.

7. Полисский Ю.Д. Некоторые вопросы выполнения сложных операций в системе остаточных классов // Электронное моделирование. - 2008. - Т. 30. - №2. - С. 115-120.

8. Ю.Д.Полисский. Алгоритм выполнения сложных операций в системе остаточных классов с помощью представления чисел в обратных кодах // Электронное моделирование. - 2014. Т.36. - № 4. - С. 117-122.

\section{METHODS OF REALIZATION OF SOME COMPLEX OPERATIONS IN THE SYSTEM OF RESIDUAL CLASSES Polissky Yu.D.}

\begin{abstract}
The operation of control systems always involves comparing the states of controlled objects that are evaluated by the corresponding values of numbers. The computing structures through which the above-mentioned operations of numerical comparison are performed are constantly required to increase the speed. Application of the system of residual classes (SRC) allows to increase the productivity of such systems. With significant advantages of the operations of comparing numbers in the SRC there are some difficulties in their implementation in this system, which require further research. The purpose of this study is the analytical review of the SRC for the implementation of complex operations of pairwise and group comparison of numbers. When comparing numbers pairwise, you need to find more or less of them. When grouping the numbers, the following tasks are solved: the definition of the maximum and minimum numbers of a group, the definition of the length of the range of the group of numbers, the determination of the position of the numbers of the group in relation to a certain fixed number, the definition of numbers inside a certain subband, the definition of the number closest to the given number, the definition the number closest to the given and the nearest
\end{abstract}


smaller to the given number. Studies of the comparison of numbers in the SRC showed that all the variety of solutions for pairwise comparison of numbers can be obtained on the basis of three approaches. First, for each of the comparable numbers, positional characteristics are calculated, after which a comparative comparison of these characteristics with the traditional methods of comparison is performed. The second approach uses the definition of the membership of numbers and their difference to the upper or lower half of the range of numbers. If the numbers belong to different halves of the range, then the larger (smaller) is the number of the upper (lower) half. If the numbers belong to one half, then, if their difference is greater than the upper half of the range, there is a decreasing number. The third approach is based on the compilation of each of the numbers of the reduced differences, resulting in the entire range of numbers is broken into a number of sub-bands, within each of which the values of the residues are the same. Next, consider the third approach to solving the problems of pairwise and group comparison of numbers based on the simultaneous representation of numbers in the forward and reverse codes with the choice of active representation in each iteration.

\section{References}

[1] Akushsky I.Ya., \& Yuditsky D.I. (1968). Mashinaia arifmetika v ostatochnikh klassakh [Machine arithmetic in the residual classes]. Moscow: Soviet Radio [in USSRian].

[2] Polissky Yu.D. (2007). Metodi sravneniia chisel v sisteme ostatochnikh klassov [Methods for comparing numbers in the system of residual classes]. Naukovii visnyk NGU - Scientific Bulletin $N M U, 1,63-66$ [in Ukrainian].

[3] Isupov K.S. (2014). Ob odnom algoritme sravneniia chisel v sisteme ostatochnikh klassov [On one algorithm for comparing numbers in the system of residual classes] Vestnik Astrakhan. Gos. Tekhn.Universiteta/ Ser. Upravlenie, vichisl, tekh. Inform. - Bulletin Astrakhan. State Tech. University. Ser. Control, Computed tech. Inform., 3, 40-49 [in Russianian]].

[4] The Knut D. (2013) Iskusstvo programmirovaniia [The art of programming] Moscow: DialecticWilliams, [in USSRian].

[5] Polissky Yu.D. (2005). Sravnenie chisel v sisteme ostatochnikh klassov [Comparison of numbers in the system of residual classes]. Trudi yubileinoi mezhdunarodnoi nauchno-technichtskoi konferencii «50 let moduliarnoi arifmeniki» - «50 years of modular arithmetic: works of the anniversary Intern. scientific and technical. Conf.». pp. 274-290.Moscow: MIET [in Russianian]].

[6] Faktorovich M.G., \& Polissky Yu.D. (1978). Ustroistvo dlia sravnenia chisel, virazhenikh v sisteme ostatochnikh klassov [A device for comparing numbers expressed in system of residual classes]. Avtorskoe svidetelstvo SSSR №608155 M.K $\Omega^{2}$ G 06 F 7/04] - Certificate of authorship USSR №608155 M.K $\pi^{2}$ G 06 F 7/04]. [in USSRian].

[7] Polissky Yu.D. (2008). Nekotorie voprosi vipolnenia slozhnikh operatsii v sisteme ostatochnikh klassov [Some questions of performing complex operations in the system residual classes]. Elektronnoe modelirovanie - Electronic Modeling, 30 (2), 115-120 . [in Ukrainian].

[8] Polissky Yu.D. (2014). Algoritm vipolnenia slozhnikh operatsii v sisteme ostatochnikh klassov [Algorithm for performing complex operations in the system of residual classes using the representation of numbers in inverse codes] Elektronnoe modelirovanie - Electronic Modeling, 36.(4), 117-122. [in Ukrainian]. 